\title{
Parameters Affecting Cellular Invasion and Escape from the Parasitophorous Vacuole by Different Infective Forms of Trypanosoma cruzi
}

\author{
RB Stecconi-Silva, WK Andreoli, RA Mortara/ ${ }^{+}$ \\ Departamento de Microbiologia, Imunologia e Parasitologia, Universidade Federal de São Paulo, Escola Paulista de Medicina, \\ Rua Botucatu 862, $6^{\circ}$ andar, 04023-062 São Paulo, SP, Brasil
}

\begin{abstract}
In this study we have examined certain aspects of the process of cell invasion and parasitophorous vacuole escape by metacyclic trypomastigotes and extracellular amastigote forms of Trypanosoma cruzi (G strain). Using Vero (and HeLa) cells as targets, we detected differences in the kinetics of vacuole escape by the two forms. Alcalinization of intercellular $p H$ influenced both invasion as well as the escape from the parasitophorous vacuole by metacyclic trypomastigotes, but not the escape kinetics of extracellular amastigotes. We used sialic acid mutants as target cells and observed that the deficiency of this molecule facilitated the escape of both infective forms. Hemolysin activity was only detected in extracellular amastigotes and neither form presented detectable transialidase activity. Invasion of extracellular amastigotes and trypomastigotes in Vero cells was affected in different ways by drugs that interfere with host cell $\mathrm{Ca}^{2+}$ mobilization. These results are in line with previous results that indicate that metacyclic trypomastigotes and extracellular amastigote forms utilize mechanisms with particular features to invade host cells and to escape from their parasitophorous vacuoles.
\end{abstract}

Key words: Trypanosoma cruzi - infective forms - parasitophorous vacuole - escape - invasion - kinetics

In order to complete its life cycle, Trypanosoma cruzi, the protozoan ethiological agent of Chagas disease must invade mammalian cells, escape the parasitophorous vacuole and then transform into amastigotes in the cytoplasm in order to grow. In this process, trypomastigotes, the classical infective forms originated from the insect vector or infected cells, and also amastigotes, derived from the extracellular transformation of trypomastigotes or by the premature release from infected cells, may invade mammalian cells in vitro as well as in vivo (Behbehani 1973, Pan 1978, Hudson et al. 1984, Ley et al. 1988, Mortara 1991).

Previous studies from our laboratory have shown that the parameters controlling the invasion of HeLa and Vero cells are highly dependent on the infective form as well as the target cell used (Procópio et al. 1998). However, both recently internalized extracellular amastigotes and metacyclic trypomastigotes could be found in compartments bearing the lysosomal marker LAMP-1 (Procópio et al. 1998). It has been shown that $\mathrm{Ca}^{2+}$ mobilization is involved in the signaling pathway leading to parasitophorous vacuole formation during trypomastigote invasion (Burleigh \& Andrews 1995). Moreover, the combined activities of hemolysin and transialidase, activated within the acidified trypomastigote parasitophorous vacuole, are believed to be crucial in providing the means for these forms to escape into the

Financial support: Fapesp, $\mathrm{CNPq}$, and Capes

${ }^{+}$Corresponding author: Fax: +55-11-5571.1095. E-mail: renato@ecb.epm.br

Received 28 February 2003

Accepted 6 August 2003 cytoplasm, that is facilitated in cells lacking proper sialylation of lysosomal glycoproteins (Andrews \& Whitlow 1989, Andrews et al. 1990, Hall et al. 1992). By contrast, little is known on the signals triggered during amastigote invasion, and the available data (Procópio et al. 1998) indicate that parasitophorous vacuole formation probably involves lysosomal recruitment. Furthermore, the mechanism by which amastigotes escape from this compartment seems to depend mostly on hemolysin (Ley et al. 1990). In the present work we have: (i) studied the kinetics of parasitophorous vacuole formation and escape of both infective forms in Vero and HeLa cells; (ii) determined the hemolytic and transialidase activities of both parasite forms; (iii) examined the effect of host cell sialic acid on parasite invasion and escape, (iv) studied the effect of compounds that interfere with calcium mobilization on the initial invasion step.

We have found significant differences between extracellular amastigotes and metacyclic trypomastigotes in relation to several of the parameters studied here.

\section{MATERIALS AND METHODS}

Cells and parasites - Vero and HeLa cells obtained from Instituto Adolpho Lutz (São Paulo, SP, Brazil) were cultivated in RPMI 1640 medium (GIBCO BRL, Grand Island, NY, US) supplemented with $10 \%$ fetal calf serum (FCS/CULTILAB, Campinas, SP, Brazil), penicillin (200 U/ $\mathrm{ml}$; Sigma Chemical, St. Louis, MO, US); streptomycin (2.5 $\mu \mathrm{g} / \mathrm{ml}$, Sigma); gentamycin ( $44 \mu \mathrm{g} / \mathrm{ml}$; Schering-Plough, São Paulo, SP, Brazil), $\mathrm{NaHCO}_{3}(2 \mathrm{~g} / \mathrm{l})$, and N-(2hidroxyetil)-piperazine-N'-(2-ethanosulfonic acid - HEPES $(2.38 \mathrm{~g} / \mathrm{l})$ in a $5 \% \mathrm{CO}_{2}$ humid atmosphere. Wild type $\mathrm{CHO}$ and sialic acid deficient Lec-2 cells (Deutscher et al. 1984) kindly provided by Sergio Schenkman from the Cell Biology division of our Department, and originally 
obtained from ATCC, American Type Culture Collection, were cultivated in $\alpha$-MEM with ribonucleosides and ribonucleotides (GIBCO) supplemented with 5\% FCS in a humid atmosphere with $5 \% \mathrm{CO}_{2}$ at $36.5^{\circ} \mathrm{C}$.

Extracellular amastigotes [of the G strain (Yoshida 1983), sylvatic phylogenetic type 1 strain (Souto et al. 1996)] were obtained from the differentiation of cellderived trypomastigotes isolated from infected Vero cells in LIT medium (Mortara 1991). Briefly cell-culture derived trypomastigotes are harvested from culture medium and resuspended in LIT medium at $37^{\circ} \mathrm{C}$ for $48 \mathrm{~h}$ (Mortara 1991). Henceforth these forms will be referred as amastigotes. Metacyclic trypomastigotes were obtained from the axenic differentiation of epimastigotes grown in LIT (liver infusion tryptose) medium containing $10 \% \mathrm{FCS}$ and $0.2 \%$ glucose at $28^{\circ} \mathrm{C}$ and purified by ionic exchange chromatography (Yoshida 1983).

Cell invasion assays - Semi-confluent cells were infected with metacyclic trypomastigotes or extracellular amastigotes in a 10:1 (parasite:cell) ratio. Excess medium was aspirated and metacyclic trypomastigotes or extracellular amastigotes suspended in complete medium, with or without drugs, were added. Cells were immediately centrifuged at $2300 \mathrm{~g}$ for $20 \mathrm{~min}$ at $37^{\circ} \mathrm{C}$. After centrifugation, unattached parasites were removed by washing the coverslips three times with RPMI without serum and seven times with sodium phosphate-buffered saline $\mathrm{pH} 7.2(\mathrm{PBS})$, both at $37^{\circ} \mathrm{C}$. Coverslips were fixed in $2 \%$ glutaraldehyde (Sigma, EM grade) in $0.1 \mathrm{M}$ sodium phosphate buffer $\mathrm{pH} 7.0$ for $1.5 \mathrm{~h}$ at room temperature. After three washings in PBS, coverslips were incubated with $0.138 \mathrm{M}$ ethanolamine, $\mathrm{pH} 8.3$ for $30 \mathrm{~min}$ at room temperature to quench excess aldehyde groups, washed with PBS, and soaked in PBS containing $0.25 \%$ gelatin and $0.05 \mathrm{M} \mathrm{NaN}_{3}$ (PGN) (Procópio et al. 1998).

After immunofluorescence incubations (described below) the invasion index was calculated according to the formula: number of parasites inside cells/ number cells infected $\mathrm{x} \%$ of infected cells, counting 100 cells in triplicate coverslips (Procópio et al. 1998).

Antibodies and immunofluorescence labeling - For invasion assay quantitations, cells fixed in $2 \%$ glutaraldehyde were incubated with monoclonal antibodies (ascitic fluids diluted 1:40 in PGN): $\mathrm{mAb} 1 \mathrm{G} 7$ against the 90 $\mathrm{kDa}$ metacyclic trypomastigote glycoprotein (Teixeira \& Yoshida 1986) or mAb 1D9, reactive against the Ssp-4 glycoprotein of amastigotes (Barros et al. 1997). After $1 \mathrm{~h}$ the coverlisps were washed 3 times with PBS and incubated $1 \mathrm{~h}$ with anti-mouse IgG-FITC conjugated (Sigma) diluted 1:50 in PGN and 10 $\mu$ M DAPI (4',6-diamidino-2-phenylindole dihydrochloride, Molecular Probes, Eugene, Oregon, US). Using this methodology only parasites outside cells are labeled with antibodies and all parasites are visible after DAPI staining. Invasion index was calculated as described above. For conventional immunofluorescence infected cells were fixed with $3.5 \%$ formaldehyde, washed and then permeabilized with PGN solution containing $0.1 \%$ saponin (Barros et al. 1997). All statistical calculations describe here were done with SigmaStat (Version 1.0, Jandel Scientific), using the T-test for significance and standard deviations for paired data sets.
Determination of parasites within parasitophorous vacuoles - Coverslips fixed and processed for conventional immunofluorescence were incubated with anti LAMP-1 antibodies (clone H4A4 anti-human LAMP-1, and clone UH3, anti-hamster LAMP-1, both developed in mouse, supernatants from DSHB, Development Studies Hybridoma Bank, Iowa, US) for $1 \mathrm{~h}$ and finally incubated $1 \mathrm{~h}$ with anti-mouse IgG conjugated to Cy3 (Sigma) diluted 1:50 in PGN and $10 \mu \mathrm{M}$ DAPI. Parasites within parasitophorous vacuoles appeared labeled with anti-LAMP1 antibodies (Hall et al. 1992). The kinetic parameters analyzed here were the half-life times for formation and escape as well as the mean time of residence within the parasitophorous vacuole, described in Fig. 1. Invasion and kinetic experiments using triplicate coverslips were performed at least three times.

Hemolysin and transialidase assays - Hemolysin (TcTOX) activity was determined as described by Andrews and Whitlow (1989), with minor modifications. Briefly, freshly collected female albino mouse erythrocytes were washed three times with isotonic PBS/ $0.1 \%$ gelatin, $0.15 \% \mathrm{CaCl}_{2}$ and $1 \mathrm{mM} \mathrm{MgCl}_{2}$. Isolated amastigotes, $\mathrm{Y}$ strain tissue-culture derived (positive controls) and $\mathrm{G}$ strain metacyclic trypomastigotes $\left(10^{8} /\right.$ $\mathrm{ml}$ ) were transferred to $10 \mathrm{mM}$ sodium acetate buffer, $\mathrm{pH}$ $5.5,0.15 \mathrm{M} \mathrm{NaCl}$, containing $1 \%$ glucose and the erythrocytes $\left(10^{7} / \mathrm{ml}\right)$. Maximum hemolytic values were obtained by treating the erythrocytes with $0.1 \%$ saponin. Released hemoglobin was measured at $540 \mathrm{~nm}$ in a Labsystems Multiskan MS (Finland) ELISA reader, in duplicate samples collected at 3, 6, and $10 \mathrm{~h}$. Transialidase activity with purified parasites was assayed as described (Schenkman et al. 1994). Briefly, the enzymatic transference of sialic acid to ${ }^{14}[\mathrm{C}]$-lactose generated ${ }^{14}[\mathrm{C}]$-sialyl-lactose that was captured in QAE-Sephadex A-25 columns, and we used Y strain tissue culture derived trypomastigotes as positive controls (Schenkman et al. 1994). These independent determinations were carried out at least three times.

Interference with cytosolic $p H$ and calcium concentrations - In order to raise cytoplasmic $\mathrm{pH}$, cells were exposed for $1 \mathrm{~h}$ at $36.5^{\circ} \mathrm{C}$, to culture medium containing $100 \mu \mathrm{M}$ chloroquine. Alternatively, cells were treated for $30 \mathrm{~min}$ with calcium ionophore A23187 or endoplasmic reticulum $\mathrm{Ca}^{2+}$ ATPase inhibitor thapsigargin (Sigma) that were used at final concentrations of $10 \mu \mathrm{M}$ and $1 \mu \mathrm{M}$ (in complete culture medium), respectively. Cells were then washed 3 times with PBS and complete medium containing the parasites was added to the flasks.

\section{RESULTS}

Formation and escape from parasitophorous vacuole by $T$. cruzi infective forms. Role of cytoplasmic $\mathrm{pH}$ - By using the centrifugation protocol developed in our laboratory, it was possible to induce a large population of parasites to rapidly associate to the target cells. Parasites were centrifuged onto the host cells, unattached parasites aspirated and the kinetics of parasitophorous vacuole formation started by monitoring the appearance of parasites labeled with anti-LAMP-1 (Fig. 1) antibodies. During a $24 \mathrm{~h}$ period, it was possible to distinguish at 
least 3 phases in the process: an initial stage of increasing numbers of labeled parasites, probably reflecting the internalization of adhered parasites; then the number of intravacuolar parasites stabilized for a period of time and then began to decrease until no labeled parasites could be detected (Fig. 2A). We will refer to these phases as parasitophorous vacuole formation, mean time of residence and escape, respectively (see Fig. 2A). Simple kinetic parameters describing the three phases were determined for both metacyclic trypomastigotes and amastigotes, in Vero and HeLa cells (Table). For the formation and escape steps, we determined the half-life of each step $\left(\mathrm{T}^{1 / 2} f\right.$ and $\mathrm{T}^{1 / 2} e$, for formation and escape, respectively). Since our determinations were performed on an hourly basis, we have arbitrarily assigned this value
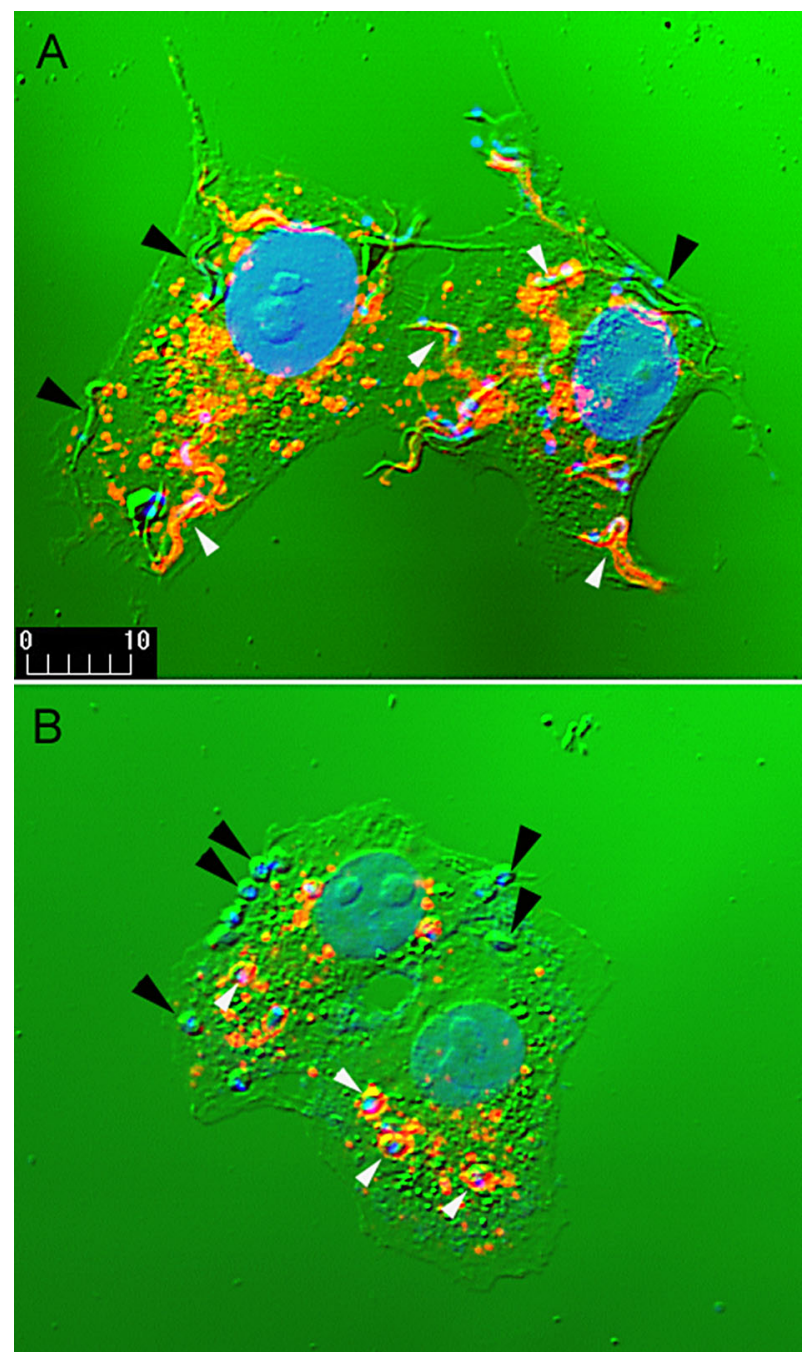

Fig. 1: determination of intracellular Trypanosoma cruzi infective forms inside and outside the parasitophorous vacuole. After centrifugation onto Vero cells, metacyclic trypomastigotes (A) or amastigotes (B) could be localized within LAMP-1 positive parasitophorous vacuoles (labeled in red, white arrowheads) or free in the cytoplasm (not labeled, black arrowheads). Differential interference contrast (green) and DNA labeling with DAPI (blue) are overlaid with LAMP-1 (red) images obtained by confocal microscopy. Magnification bar $=10 \mu \mathrm{m}$ for the mean error for each experimental point. The parameters determined for metacyclic trypomastigotes (Fig. 2B) and amastigotes (Fig. 2C) shown in the Table, indicate that there were no significant differences between the two forms with either target cell. Although no significant difference in the kinetics of formation between the two target cells or infective forms were observed, we consistently found that amastigotes tended to escape faster from the parasitophorous vacuole since after $12 \mathrm{~h}$, more than $90 \%$ of the amastigotes were already free in the cytoplasm, whereas at this time $20-30 \%$ of the metacyclic forms were still labeled for anti-LAMP-1 antibodies (Fig. 2A, B). The mean time of residence within the parasitophorous vacuole was usually shorter for metacyclic forms when compared to amastigotes, regardless of the target cell used. The escape phase was comparable between the two infective forms and there were no major differences between the two target cells. When the cytoplasmic $\mathrm{pH}$ of Vero cells was raised with chloroquine the time of residence of metacyclic trypomastigotes increased from 4 to about $10 \mathrm{~h}$, whereas no effect on the kinetics of amastigotes was observed (Fig. 2B, C and Table). Interestingly, the kinetics of vacuolar escape $T^{1 / 2} e$ by either infective form was not affected by raising cytoplasmic $\mathrm{pH}$ (Table). Chloroquine treatment only inhibited the invasion of metacyclic trypomastigotes (Fig. 3).

Role of host cell sialic acid on parasite invasion and vacuole escape - We used $\mathrm{CHO}$ mutant Lec- 2 cells that are deficient in sialic acid in order to examine the role of host cell sialic acid on parasite invasion and escape from the parasitophorous vacuole. The susceptibility to invasion of Lec-2 cells by both $T$. cruzi infective forms was essentially the same as that of the parental $\mathrm{CHO}$ line (Fig. 4 A, B). We then examined the escape by the two forms by counting parasites inside the parasitophorous vacuole after 3 and $6 \mathrm{~h}$ post-infection and verified an efficient escape from the parasitophorous vacuoles of both forms in Lec-2 cells indicated by a significant loss of labeling with anti-LAMP1 (Fig. 4 C, D).

Transialidase and hemolysin activities of metacyclic trypomastigotes and amastigotes - The transialidase activity of isolated parasites was negligible for both forms when compared to positive controls Y strain tissue culture trypomastigotes (data not shown). When we assayed isolated parasites for hemolytic (TcTOX) activity, we could only detect hemolysis by amastigotes that after $12 \mathrm{~h}$ caused the rupture of $90 \%$ red blood cells. By contrast, no lytic activity could be detected in metacyclic trypomastigotes (not shown).

Interference with host calcium only affects metacyclic trypomastigote invasion - Compounds that interfere with host cell $\mathrm{Ca}^{2+}$ mobilization may interfere with parasite invasion and we have confirmed these observations by using thapsigargin and calcium ionophore A23187. Inhibition of endoplasmic reticulum calcium ATPase by thapsigargin inhibited both Vero (Fig. 5A) and HeLa (not shown) cell invasion by metacyclic trypomastigote. Less pronounced inhibitory effect was obtained with A23187 (Fig. 5A). Host cell invasion by amastigotes was not affected by either compound (Fig. 5B). 


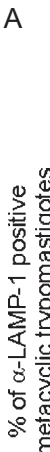

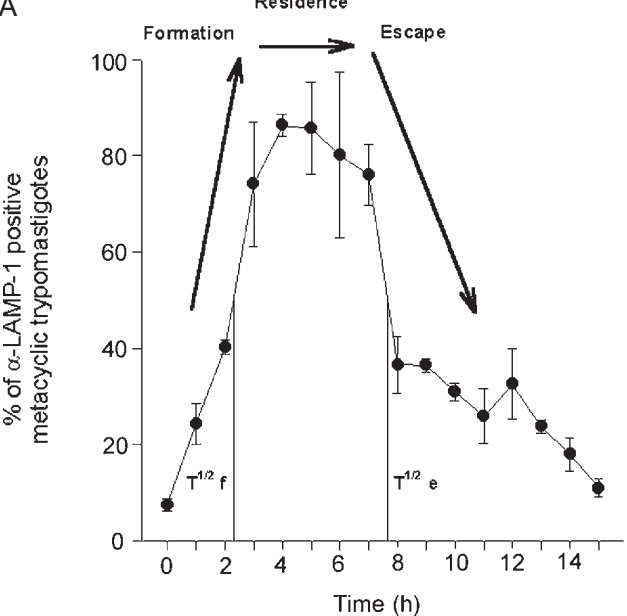

B

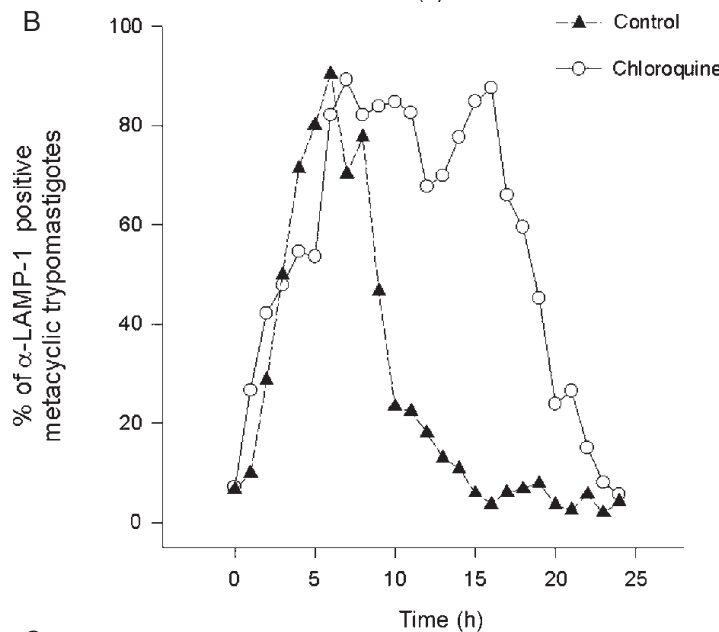

C

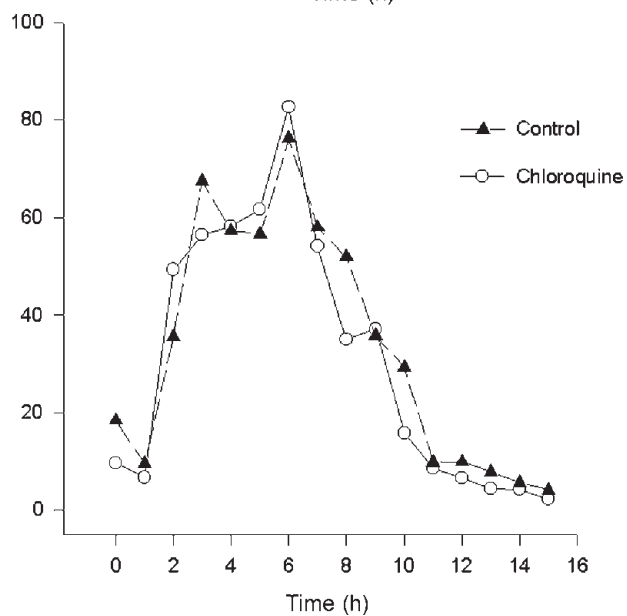

Fig. 2: chloroquine increases the time of residence of Trypanosoma cruzi metacyclic trypomastigotes in the parasitophorous vacuole. A: kinetics of T. cruzi parasitophorous vacuole formation in Vero cells determined by the percentage of anti-LAMP-1 positive parasites at different times. The sequential steps of the process are indicated: formation, residence and escape. The half-lives of the formation and escape steps are also indicated; B: data obtained in the presence of $100 \mu \mathrm{M}$ chloroquine are represented by open circles: metacyclic trypomastigotes remain up to $6 \mathrm{~h}$ more in the parasitophorous vacuole when compared to the controls, represented by closed triangles; $\mathrm{C}$ : the overall kinetics of amastigote parasitophorous vacuole formation, residence and escape, are not affected by chloroquine (controls represented by closed triangles, $100 \mu \mathrm{M}$ chloroquine by open circles).
TABLE

Kinetic parameters of parasitophorous vacuole establishment by Trypanosoma cruzi infective forms in cultured mammalian cells (see 2A)

\begin{tabular}{lccc}
\hline & HeLa & Vero & $\begin{array}{c}\text { Vero+100 mM } \\
\text { Chloroquine }\end{array}$ \\
\hline $\mathrm{T}^{1 / 2}$ Formation/Meta $^{a}$ & 3 & 2 & 2 \\
Residence/Meta $^{b}$ & 4 & 4 & 10 \\
$\mathrm{~T}^{1 / 2}$ Escape/Meta $^{c}$ & 4 & 3 & 3 \\
$\mathrm{~T}^{1 / 2}$ Formation/Ama & 2 & 2 & 2 \\
Residence/Ama $^{1 / 2}$ Escape/Ama & 5 & 5 & 4 \\
$\mathrm{~T}^{1 / 2}$ & 3 & 3 & 4 \\
\hline
\end{tabular}

a: $\mathrm{T}^{1 / 2}$ formation: half-life for parasitophorous vacuole formation. As indicated in the text, a mean error of $\pm 1 \mathrm{~h}$ is assumed for each determination; $b$ : mean time of residence; $c$ : $T^{1 / 2}$ escape: half-life for parasitophorous escape

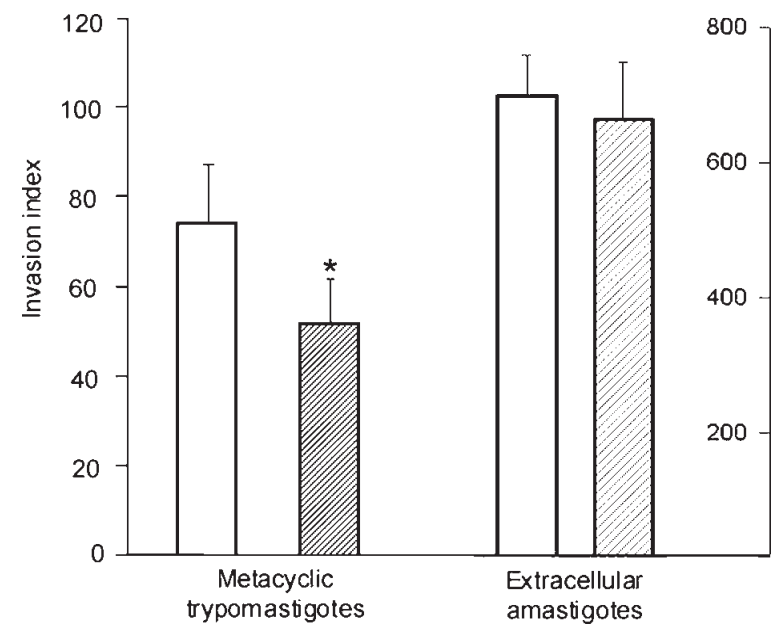

Fig. 3: cytoplasmic alkalinization of Vero cells with chloroquine only inhibits metacyclic trypomastigote invasion. Vero cells were incubated with $100 \mu \mathrm{M}$ chloroquine (represented by hatched bars) and exposed to Trypanosoma cruzi infective forms. Invasion indexes were determined as indicated in the text. Bars denote mean three experiments with standard deviations and *indicates that $\mathrm{p} \leq 0.05$ relative to the controls (represented by open bars).

\section{DISCUSSION}

We have shown here that different infective forms of T. cruzi exhibit distinct behavior throughout the process of cell invasion, parasitophorous vacuole formation and escape. Even though chloroquine inhibited metacyclic trypomastigote invasion (Fig. 3), the overall effect of raising cytoplasmic $\mathrm{pH}$ was not detectable in the kinetics of vacuole formation (Fig. 2) but dramatically affected their escape, increasing the mean residence by at least a factor of two, whereas not affecting the parameters of amastigote transit, suggesting that the mechanisms engaged for parasitophorous membrane disruption is stage-specific. Of the two known components involved, hemolysin and transialidase, (Andrews \& Whitlow 1989, Andrews et al. 1990, Hall et al. 1992) only hemolysin activity could be detected in amastigotes whereas in metacyclic trypomastigotes none of the activities could be detected. 

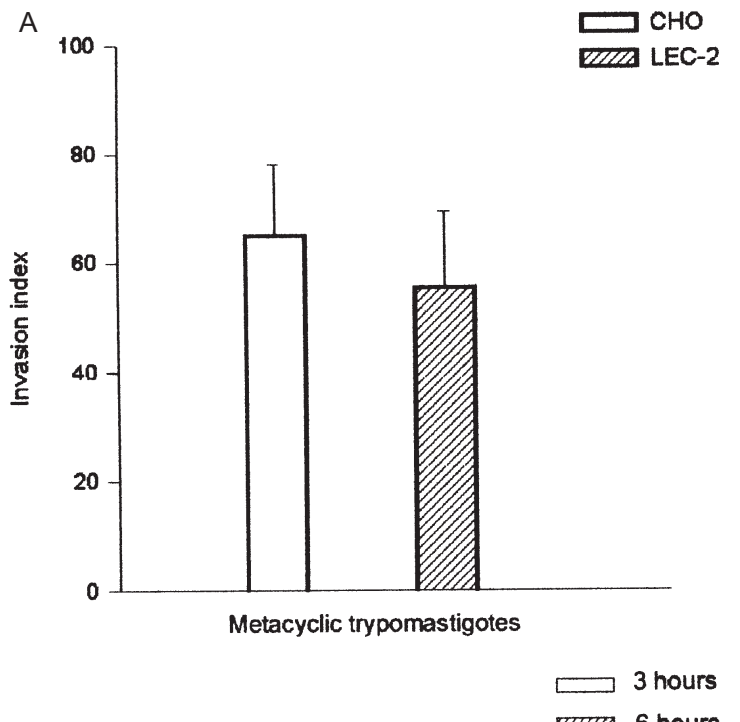

C

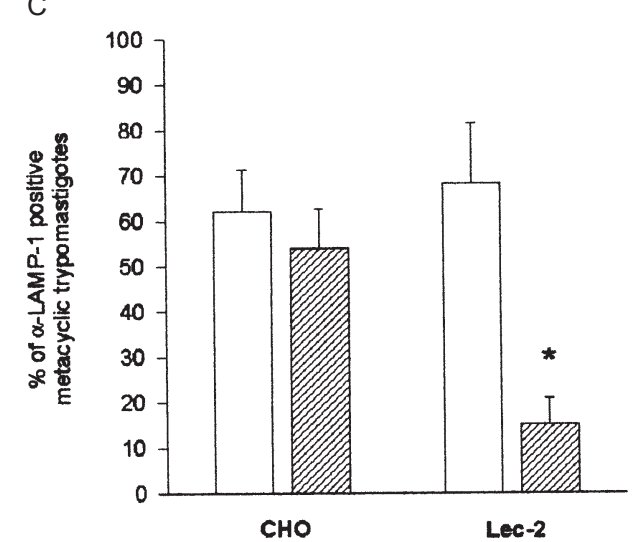

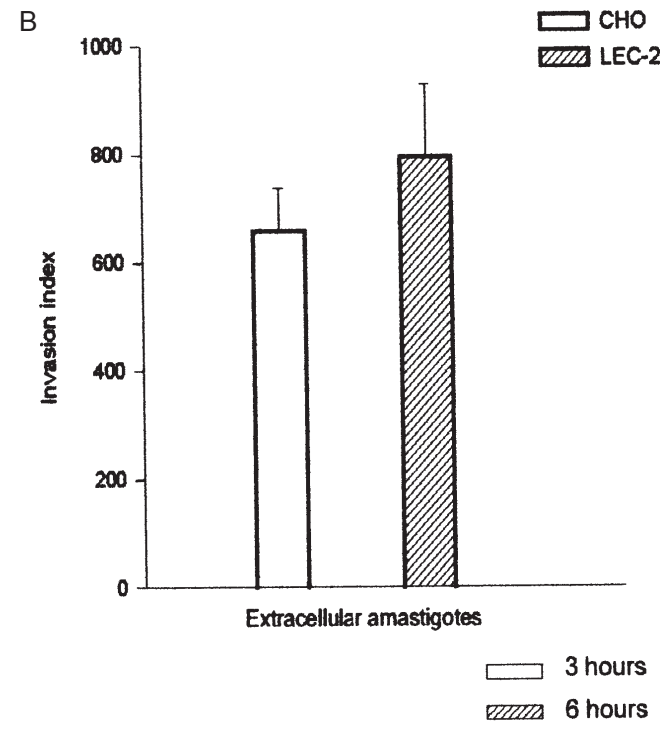

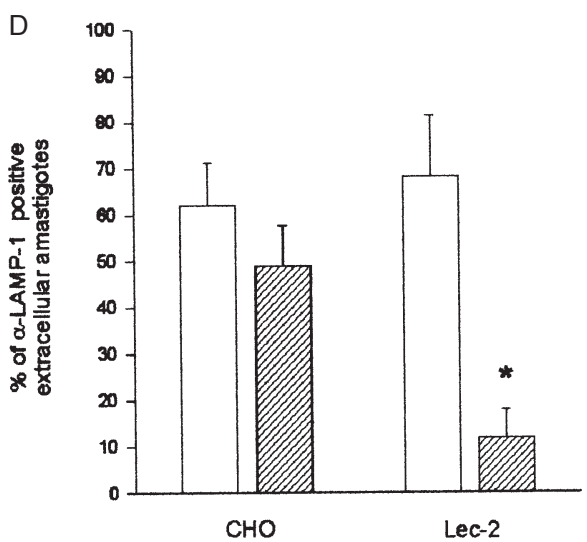

Fig. 4: reduced expression of sialic acid on host cells affects parasitophorous vacuole escape but not invasion by Trypanosoma cruzi infective forms. A, B: CHO and sialic acid-deficient Lec-2 mutant cells were infected with metacyclic trypomastigotes (A) or amastigotes (B) and invasion indexes determined as described in Materials and Methods; C, D: the percentages of remaining LAMP-1 labeled parasites were determined after 3 and $6 \mathrm{~h}$. Bars denote mean three experiments with standard deviations and *indicates that $\mathrm{p} \leq 0.05$ relative to the $\mathrm{CHO}$ cell controls. Note that the reduction in anti-LAMP-1 labeling, compatible with parasitophorous vacuole escape, is significant for both infective forms in Lec-2 cells.

Whereas hemolysin activity has been shown to be $\mathrm{pH}$ sensitive and hence inhabitable by $\mathrm{pH}$ elevation (Ley et al. 1990) this activity is apparently lacking in metacyclics of the $\mathrm{G}$ as well as of CL (Andreoli \& Mortara 2003) strains. Unexpectedly, amastigotes of the G strain (this study) as well as of the Y strain (Ley et al. 1990) that express $\mathrm{pH}$ sensitive hemolysin are not affected by raising the cytoplasmic $\mathrm{pH}$ of the target cell (Fig. 2). These apparently contradictory findings thus suggest that other unknown mechanisms might either operate or be induced once the parasites are trapped within the parasitophorous vacuole. Among other factors, the tightness of this compartment might be a relevant one (Lopez et al. 2002). The kinetics of metacyclic trypomastigote escape from the parasitophorous vacuole (Fig. 2A, B) also suggests that there may be two distinct populations of parasites: a relatively fast escaping group that leaves the vacuole from 7 to $8 \mathrm{~h}$ and another set that remains in the vacuole for more that
$5 \mathrm{~h}$ slowly leaving the vacuole between 12 to $15 \mathrm{~h}$ post invasion.

Although the presence of sialic acid on host cell glycoproteins appears to enhance trypomastigote invasion (Schenkman et al. 1993) and may also protect the parasitophorous membrane from the action of the above mentioned required factors for parasite escape (Hall et al. 1992), the invasion step by the two infective forms does not seem to be affected by host cell sialic acid (Fig. 4). This observation contrasts to the observation that $Y$ (a type II strain) tissue-culture derived trypomastigotes appear to preferentially invade sialylated target cells (Schenkman et al. 1993). However, in salic acid deficient Lec-2 cells, the escape of both infective forms studied here is facilitated by sialic acid deficiency (Fig. 4), as previously described for Y strain tissue-culture derived trypomastigotes (Hall et al. 1992). Taken together, these findings reinforce the notion that there may be yet 

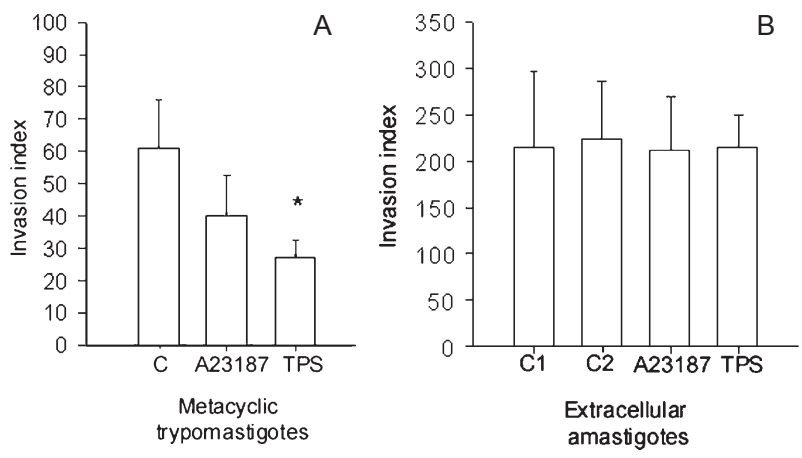

Fig. 5: drugs that affect host cell free calcium concentration only inhibit metacyclic trypomastigote invasion. Vero cells were incubated for $30 \mathrm{~min}$ with $10 \mathrm{mM}$ calcium ionophore A23187 or 1 $\mathrm{mM}$ thapsigargin (TPS), and subjected to invasion by metacyclic trypomastigotes (A) or amastigotes (B). Bars denote mean three experiments with standard deviations and *indicates $\mathrm{p} \leq 0.05$ relative to the controls. $\mathrm{C}$ is the control for $\mathrm{A}$, and in $\mathrm{B}, \mathrm{C} 1$ and $\mathrm{C} 2$ are the controls carried out for each drug.

unknown mechanisms involved in the escape of these infective forms, particularly of metacyclic trypomastigotes, into the cytoplasm.

It has recently been shown that $T$. cruzi exists as at least two distinct phylogenetic lineages in nature (Souto et al. 1996). The G strain used in this study belongs to the sylvatic type I and it has recently been show that invasion mechanisms of metacyclic trypomastigotes of the two lineages are highly divergent (Neira et al. 2002). We have confirmed here that the requirement for host cell calcium mobilization is also dependent on the infective form since the invasion of Vero cells by amastigotes, in contrast to metacyclic trypomastigotes, is not affected by agents that modify host cell free calcium concentration (Fig. 5). We are currently undertaking studies to comprehensively compare the invasion and escape mechanisms used by infective forms of the two phylogenetic lineages.

In conclusion, we have shown that two distinct infective forms of the parasite display distinct behavior that probably reflect the engagement of specific mechanisms in the process of host cell invasion and parasitophorous vacuole escape.

\section{ACKNOWLEDGEMENTS}

To Nobuko Yoshida for her critical review of the manuscript.

\section{REFERENCES}

Andreoli WK, Mortara RA 2003. Acidification modulates the traffic of Trypanosoma cruzi trypomastigotes in Vero cells harboring Coxiella burnetti vacuoles. Int J Parasitol 33: 183195.

Andrews NW, Whitlow MB 1989. Secretion by Trypanosoma cruzi of a hemolysin active at low pH. Mol Biochem Parasitol 33: 249-256.

Andrews NW, Abrams CK, Slatin SL, Griffiths G 1990. A T. cruzi-secreted protein immunologically related to the complement component C9: evidence for membrane poreforming activity at low $\mathrm{pH}$. Cell 61: 1277-1287.

Barros HC, Verbisck NV, Silva S, Araguth MF, Mortara RA
1997. Distribution of epitopes of Trypanosoma cruzi amastigotes during the intracellular life cycle within mammalian cells. J Eukaryot Microbiol 44: 332-344.

Behbehani K 1973. Developmental cycles of Trypanosoma (Schyzotrypanum) cruzi (Chagas, 1909) in mouse peritoneal macrophages in vitro. Parasitology 66: 343-353.

Burleigh BA, Andrews NW 1995. The mechanisms of Trypanosoma cruzi invasion of mammalian cells. Annu Rev Microbiol 49: 175-200.

Deutscher SL, Nuwayhid N, Stanley P, Briles EI, Hirschberg CB 1984. Translocation across Golgi vesicle membranes: a $\mathrm{CHO}$ glycosylation mutant deficient in CMP-sialic acid transport. Cell 39: 295-299.

Hall BF, Webster P, Ma AK, Joiner KA, Andrews NW 1992. Desialylation of lysosomal membrane glycoproteins by Trypanosoma cruzi: A role for the surface neuraminidase in facilitating parasite entry into the host cell cytoplasm. $J$ Exp Med 176: 313-325.

Hudson L, Snary D, Morgan SJ 1984. Trypanosoma cruzi: continuous cultivation with murine cell lines. Parasitology 88: 283-294.

Ley V, Andrews NW, Robbins ES, Nussenzweig V 1988. Amastigotes of Trypanosoma cruzi sustain an infective cycle in mammalian cells. J Exp Med 168: 649-659.

Ley V, Robbins ES, Nussenzweig V, Andrews NW 1990. The exit of Trypanosoma cruzi from the phagosome is inhibited by raising the $\mathrm{pH}$ of acidic compartments. J Exp Med 171: 401-413.

Lopez M, Huynh C, Andrade LO, Pypaert M, Andrews NW 2002. Role for sialic acid in the formation of tight lysosomederived vacuoles during Trypanosoma cruzi invasion. Mol Biochem Parasitol 119: 141-145.

Mortara RA 1991. Trypanosoma cruzi: amastigotes and trypomastigotes interact with different structures on the surface of HeLa cells. Exp Parasitol 73: 1-14.

Neira I, Ferreira AT, Yoshida N 2002. Activation of distinct signal transduction pathways in Trypanosoma cruzi isolates with differential capacity to invade host cells. Int J Parasitol 32: 405-414.

Pan SCT 1978. Trypanosoma cruzi: In vitro interactions between cultured amastigotes and human skin-muscle cells. Exp Parasitol 45: 274-286.

Procópio DO, Silva S, Cunningham CC, Mortara RA 1998. Trypanosoma cruzi: effect of protein kinase inhibitors and cytoskeletal protein organization and expression on host cell invasion by amastigotes and metacyclic trypomastigotes. Exp Parasitol 90: 1-13.

Schenkman RPF, Vandekerckhove F, Schenkman S 1993. Mammalian cell sialic acid enhances invasion by Trypanosoma cruzi. Infect Immun 61: 898-902.

Schenkman S, Chaves LB, Pontes de Carvalho LC, Eichinger D 1994. A proteolytic fragment of Trypanosoma cruzi transsialidase lacking the carboxyl-terminal domain is active, monomeric, and generates antibodies that inhibit enzymatic activity. J Biol Chem 269: 7970-7975.

Souto RP, Fernandes O, Macedo AM, Campbell DA, Zingales B 1996. DNA markers define two major phylogenetic lineages of Trypanosoma cruzi. Mol Biochem Parasitol 83: 141-152.

Teixeira MMG, Yoshida N 1986. Stage-specific surface antigens of metacyclic trypomastigotes of Trypanosoma cruzi identified by monoclonal antibodies. Mol Biochem Parasitol 18: 271-282.

Yoshida N 1983. Surface antigens of metacyclic trypomastigotes of Trypanosoma cruzi. Infect Immun 40: 836-839. 